\title{
Analysis of moist air unwatering by adsorbent in fluidized bed for thermal power stations units conservation
}

\author{
Sergey Goldaev ${ }^{1}$ and Alisher Khushvaktov ${ }^{2, a}$ \\ ${ }^{1}$ Dr. Sci. (Phys. -Math.) National Research Tomsk Polytechnic University, 634050 Tomsk, Russia \\ ${ }^{2}$ Postgraduate, Tajik Technical University, 734042 Dushanbe, Tajikistan
}

\begin{abstract}
Within the model of adsorption unit with a fluidized bed of silica gel the necessary weight to place it inside the aggregates of TPP water vapor path was determined. Fluidized mode is efficient due to the developed mass transfer surface. On the other hand, it is power-consuming and more complex in structure than the embodiment proposed by the authors with car-tridges filled with silica gel. At the same time the adsorbers size will increase, but it is simplifies and reduces the cost of construction due to the desorption stage rejection.
\end{abstract}

Maintaining the required humidity in closed volumes is performed using various adsorbents $[1,2]$. When the relative moisture content of the medium $(\varphi)$ is less than $55 \ldots 60 \%$, small pore silica with bulk density $\rho_{s} \sim 700 \mathrm{~kg} / \mathrm{m}^{3}$ is often used. In the range of $\varphi \approx 60 \ldots 90 \%$ medium pore gel is effective [1].

Silica gels moisture saturation process under static conditions is characterized by a low speed. At room temperature the duration of small porous silica gels complete saturation is -40 hours, the medium porous -50 hours, and macro porou -75 hours [2].

Desorption of the absorber, which is necessary in a number of technologies that require a large air intake or its purification in autonomous conditions (orbital stations, submarines) is energy-consuming step [3].

In [6], based on the thermodynamic approach was obtained that for drying of air with the relative concentration $\varphi_{1}=80 \%$ inside the units of $100 \mathrm{~m}^{3}$ capacity by the silica gel with saturation capacity $g_{p s}=0.2$, the maximum value of the required mass of adsorbent was about $9.5 \mathrm{~kg}$. According to a survey of the silica gel market in the CIS [7] on the November 2013 the price of $1 \mathrm{~kg}$ of silica gel technical averaged $50 \mathrm{P}$ and $140 \mathrm{P}$ zeolite. Consequently, the cost of its acquisition and use are small. Silica gel can be placed in different places in the form of the absorption tank rounds [3]. If you need to drain a fixed amount of air and adsorbent produced on an industrial scale has a low price, it is the authors' opinion, it is permissible to implement disposable absorbing vegetation cartridges loaded with silica gel or zeolite. If necessary, this procedure is repeated several times.

The calculation results of dimensionally-weight adsorbers characteristics of medium pressure air separation plants filled by zeolite $\mathrm{NaX}$ showed that by using of $48 \mathrm{~kg}$ zeolite mass for the adsorption time 1.8 hours $0.757 \mathrm{~kg}$ are able to absorb water vapor [6]. These values are in qualitative agreement with the above data for silica gel.

\footnotetext{
${ }^{a}$ Corresponding author: alisher1001@mail.ru
}

This is an Open Access article distributed under the terms of the Creative Commons Attribution License 4.0, which permits unrestricted use, distribution, and reproduction in any medium, provided the original work is properly cited. 
One of the main characteristics of an adsorbent is its dynamic activity. It is known that the value of this parameter by water vapor in the zeolite is about 1.5 times greater than for silica gel [2], so the estimations can be used physic-chemical characteristics of zeolites better reported in the literature.

Adsorption processes may be implemented in the layers of particulate adsorbent, which are motionless or moving or in a fluidized state, in which the mechanical interaction of the adsorbent particles to each other and with internal apparatus surfaces $[1,2]$.

Practical purpose of the adsorption process calculating is to determine the concentration of the target component in the carrier gas at the outlet from the adsorbent bed.

The characteristics calculating algorithm for fluidized layer described in [10]. However, in the example the calculations results of one option are presented which does not allow analyzing the possibilities of such option for this circuit.

In the apparatus operation with the adsorbent fluidized bed (FB) a significant mixing of the particulate material adjustment layer having a value of $60 \ldots 100 \mathrm{~mm}$ is occurred, which provides a satisfactory fluidization [7]. It is assumed that complete adsorbent mixing is carried out, which corresponds to a constant value of the average mining degree throughout the volume of the adsorbent layer.

In most industrial plants the adsorption processes are carried out in a FB apparatus with continuous feed and continuous discharge of the adsorbent which is effective in components capturing target from exhaust gas streams of large flow.

In large-scale production the implementation of the adsorption processes in fluidized continuous fluid bed is efficient.

To estimate the minimum adsorbent amount required for the air dehumidify by the above-proposed scheme the ratios for continuous adsorption can be used.

An approximate calculation of continuous adsorber characteristics is based on the general equation of mass transfer, which includes the material balance Eq. (7)

$$
W_{b}\left(C_{0}-C_{k}\right)=W_{a}\left(a_{m d}-a_{n}\right)=\beta_{0} \Delta C_{m d} F,
$$

where $a_{m d}, a_{n}$ - average mining degree of dispersed adsorbent in the FB volume and at the outlet thereof; $W_{c}, W_{a}$ - consumption of carrier gas and adsorbent; $F$ - total surface area of the particles in the bulk layer.

Average in height layer driving concentration difference of adsorptive is calculated as follows:

$$
\Delta C_{m d}=\left(C_{0}-C_{k}\right) / \ln \left[\frac{C_{0}-C_{s}\left(a_{m d}\right)}{C_{k}-C_{s}\left(a_{m d}\right)}\right],
$$

where $C_{s}\left(a_{m d}\right)$ - adsorbate concentration in the gas, is in equilibrium with its average content in the adsorbent particles; in conditions of complete particles mixing in the FB it is constant, but unknown quantity.

In the draft calculation version such parameters are assumed known: $W_{b}, C_{0}, C_{k}$ and $a_{n}$. The mass transfer coefficient is determined by the criterion equation. For example, the results of experiments on the continuous adsorption of water vapor from the air in multi-unit with vertical FB zeolites are summarized by the following expression

$$
\frac{\beta_{0 v} d_{a}^{2}}{D_{w}}=4,0\left(\frac{W_{a} d}{D_{w}}\right)^{0,70}\left(\frac{C_{m x}}{C_{0}}\right)^{0,35}\left(\frac{\beta^{2}}{B T^{2}}\right)^{1,1},
$$

where $d_{a}$ - the adsorbent particle diameter; $C_{m x}$ - the limiting concentration of the target component in the adsorbent; $D_{w}$ and $C_{0}$ - diffusion coefficient and the initial concentration of the adsorbate vapor in the air. 
It is required to determine the total surface amount $F$ of adsorbent particles in the layer, which may provide a predetermined range of concentrations in the gas phase. Adsorbent flow $W_{a}$ is determined from additional considerations.

In a simplified embodiment, $W_{a}$ is used for calculating the minimum value at which the flow rate of the adsorbent $W_{a m n}$ at the output of the power unit mining adsorbent is in equilibrium with a given value of the input concentration in the gaseous phase

$$
W_{\text {amn }}\left(a_{s}-a_{n}\right)=W_{b}\left(C_{0}-C_{k}\right) .
$$

On the basis of experience with this type of adsorber an actual adsorbent flow rate taken more than the minimum of $10 \ldots 30 \%$

$$
W_{a}=k_{z} W_{a m n} .
$$

Illustrate the application of the methodology described in the following example from the monograph [6].

Adsorber with FB designed to absorb the water vapor from the air stream with a flow rate $W_{b}=0.50 \mathrm{~m}^{3} / \mathrm{s}$. The initial water vapor concentration is $C_{0}=3.8 \cdot 10^{-3} \mathrm{~kg} / \mathrm{m}^{3}$, final $-C_{k}=0.04$. $10^{-3} \mathrm{~kg} / \mathrm{m}^{3}$, their affinity coefficient $\beta=2.53$. Saturated water vapor concentration $\left(C_{s}\right)$ at $20 \mathrm{C}$ is equal to $17.2 \cdot 10^{-3} \mathrm{~kg} / \mathrm{m}^{3}$. The water content of the adsorbent source is $a_{n}=0.5 \mathrm{~kg} / \mathrm{m}^{3}$. Adsorbent particle density is $\rho_{a}=1200 \mathrm{~kg} / \mathrm{m}^{3}$, the diameter is $d_{a}=2.0 \cdot 10^{-3} \mathrm{~m}$, the zeolite structure constant $B=5.5 \cdot 10^{-8} 1 / \mathrm{K}^{2}$. The diffusion coefficient of water vapor in air $D_{w}=2.4 \cdot 10^{-5} \mathrm{~m}^{2} / \mathrm{s}$. Adsorption isotherm at $20 \mathrm{C}$ is assumed close to linear

$$
a_{s c}=K_{a}\left(C / C_{s}\right),
$$

where $K_{a}=679,4$ - approximation ratio of the zeolite, found from the intermediate calculations results of the considered example.

The air drying process organization in the mode of filtering it through the FB adsorbent gives a possibility to consider the results like a "from below" estimation, for example, the mass of adsorbent.

Reynolds number for the air stream passing through the FB is calculated from the Eq. (6)

$$
\operatorname{Re}_{b}=\frac{\operatorname{Ar}_{b} \cdot \varepsilon^{4,75}}{18+0,61 \sqrt{\operatorname{Ar}_{b} \cdot \varepsilon^{4,75}}},
$$

where $\varepsilon$-layer porosity is taken equal to $0.5 ; \mathrm{Ar}_{b}-\mathrm{Archimedes}$ criterion

$$
\operatorname{Ar}_{b}=\frac{g d^{3}}{v_{b}^{2}} \frac{\left(\rho_{T}-\rho_{b}\right)}{\rho_{b}}
$$

here $\rho_{b}, v_{b}-$ the density and kinematic viscosity of air at $20^{\circ} \mathrm{C}$.

In the improved method of calculating the required thermal properties of air the interpolation dependencies from the handbook [6] were used.

The average velocity of the gas at the full section of the layer

$$
w_{b}=v_{b} \cdot \operatorname{Re}_{b} / d_{T} .
$$

Diameter cylindrical shape of the cabinet

$$
D_{a p}=\sqrt{4 V_{b} /\left(\pi w_{b}\right)} .
$$

Minimum adsorbent flow rate is determined by the Eq. (4).

$$
W_{T m n}=W_{b} \frac{\left(C_{0}-C_{k}\right)}{\left[a_{s}\left(C_{k}\right)-a_{n}\right]},
$$

where according to the adsorption isotherm (6) $a_{s_{c}}\left(C_{k}\right)=1,58 \mathrm{~kg} / \mathrm{m}^{3}$. 


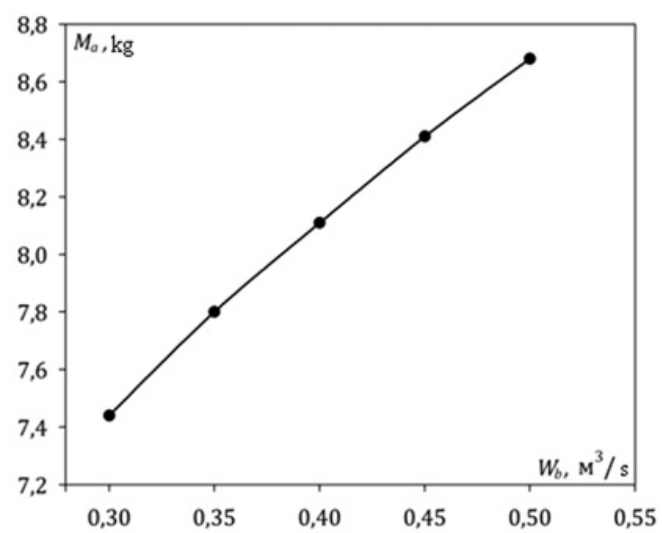

Figure 1. Dependence of the zeolite mass from air flow rate.

Taking into account the maximum stock value of adsorbent flow:

$$
V_{T}=1,3 V_{T m n} .
$$

The volumetric mass transfer coefficient at the outlet of the device is determined by the approximation Eq. (3).

The average concentration of moisture absorbed by the adsorbent was calculated from the material balance equation

$$
a_{m d}=a_{n}+\frac{V_{b}}{M_{T}}\left(C_{0}-C_{k}\right) .
$$

According to the adsorption isotherm $C_{S_{c}}\left(a_{m d}\right)=3,37 \cdot 10^{-5} \mathrm{~kg} / \mathrm{m}^{3}$.

Average driving concentration difference on the gas phase was calculated according to the above formula. According to Eq. (1) the volume and mass of adsorbent in FB were determined:

$$
V_{a}=V_{b}\left(C_{0}-C_{k}\right) / \beta_{0 v} \Delta C_{m d} ; \quad M_{a}=\rho_{a} V_{a}
$$

The volume and layer height of the FB:

$$
V_{s l}=V_{a} / \varepsilon ; \quad H_{c l}=4 V_{c l} /\left(\pi D_{a p}^{2}\right) .
$$

The described technique has been implemented in Turbo Pascal. The above input data and "manual" calculation results from the monograph [7] are used to test the compiled program.

During the parametric analysis the dependence of FB mass and the dimensions from a number of input parameters has been revealed.

Reducing the air volume flow $W_{b}$ from 0.5 to $0.3 \mathrm{~m}^{3} / \mathrm{s}$ resulted in a reduction in the mass of the zeolite from 8.7 to $7.4 \mathrm{~kg}$ (Fig. 1).

As can be seen, for drying sufficiently high air flow rate comparable to flow rates in industrial settings, several kilograms of zeolite are required. Therefore we can use the above-proposed conversion factor, and assume that the mass of silica gel will exceed by 1.5 times the zeolite mass values shown in Figs. 1 and 2.

Figure 2 shows the results of varying the initial concentration of water vapor containing in the air to the required adsorbent weight. Such curve behavior can be explained by the dependence of the adsorbent volume from mass-transfer coefficient. According to (8) $W_{a} \sim 1 / \beta_{0 v}$, and the value $\beta_{0_{v}}$, calculated by interpolation formula (3) increases from 440 (for $\mathrm{C}_{0}=0.0038 \mathrm{~kg} / \mathrm{m}^{3}$ ) to $6121 / \mathrm{s}\left(\mathrm{kg} / \mathrm{m}^{3} \mathrm{C}_{0}=0.0098\right.$ ). 


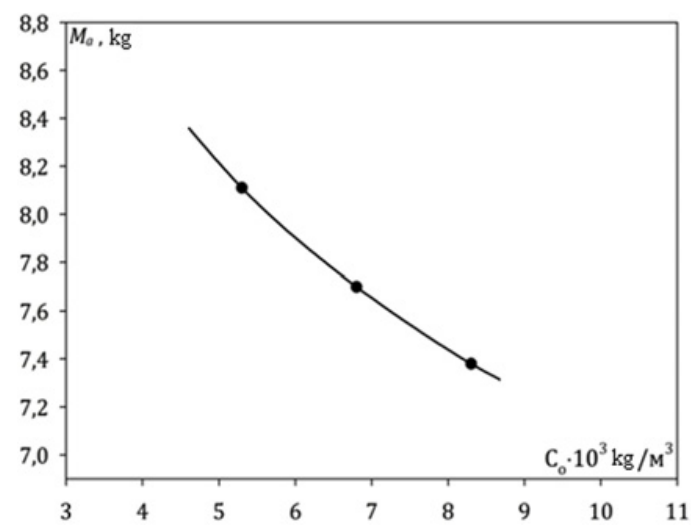

Figure 2. Dependency of the zeolite weight from initial water vapor concentration in the air.

Thus, in the model framework of adsorption unit with FB the necessary silica gel weight is determined, which is $7 \ldots 12 \mathrm{~kg}$ and does not require special tools for its placement within the TPS units. FB mode is efficient due to the developed mass transfer surface. On the other hand, it is powerconsuming and more complex in structure than the embodiment proposed by the authors with cartridges filled with silica gel. At the same time the adsorbers size will increase, but it is simplifies and reduces the cost of construction due to the desorption stage rejection.

\section{References}

[1] Serpionova E. N., Promishlennaya adsorbciya gazov i parov [Industrial adsorption of gases and vapors]. Moscow: "Hi school", 1969

[2] N. Kelcev, Fundamentals of adsorption technology. Moscow: "Chemistry", (1984)

[3] S. Mozgovoi, Extended abstract of PhD dissertation. Moscow: (2001)

[4] S. Goldaev, A. Khushvaktov, Energy: Efficiency, reliability, safety: material works XIX Russian Sci. Tech. Conf. 2. 386-389 (2013)

[5] Alekseev V. P., Vainshtain G.E., Gerasimov P. V. Raschet i modelirovanie apparatov kriogennyh ustanovok [Calculation and simulation of cryogenic devices] Energoatomizdat. Leningrad. 1987

[6] P. Romankov, V. Frolov, Mass transfer processes of chemical technology, Leningrad: "Chemistry", (1990)

[7] P. Bazhan, V. Silvestorov, Handbook of heat transfer devices, Moscow. (1989) 